\title{
Do Contrato Estimatório
}

(Notas para um estudo)

S. Soares de Faria

1 - Johhnnis Voet, no seu comentário Ad Pandectas, assim define o contrato estimatório: "Contractus aestimatorius est innominatus, bonae fidei, quo, res alteri aestimata vendenta traditur, ea lege, ut vel pretium, vel ipsa res incorrupta restituatur" (1).

$\mathrm{Na}$ definição apresentada, além de definir o contrato, - notavel pandectista faz a sua qualificação, filiando-o à espécie a que pertence.

Dernburg e outros pandectistas limitam-se à pura caracterização: é um negócio pelo qual é entregue uma cousa a um revendedor para que a venda, com o pacto de restituir ou a própria cousa ou o preço de estima convencionado. Die ist ein Geschäft, bei welchem dem Trödler eine Sache zum Verhandeln unter der Vereinbarung übergeben wird, dass er entweeder die Sache oder einem bestimmten Schätzumgpreis zurückgebe". Ou ainda "Beim Trödelvertrag übergibt man eine Sache dem Trödler zum verkaufe unter einer Taxe mit der Vereinbarung, dass er di Sache oder die Taxsumme zürückerstatte" (3).

LEONARD diz que, no contrato estimatório, se consígna uma cousa estimada, com a autorização de vendê-la, por

(1) Lib. XIX, III, vol. III, 190.

(2) Römisches Recht, vol. II, § 263, pg. 550.

(3) Id. id. $\S 377$, pg. 803 . 
meio da qual o que a recebe consegue o direito de adquiríla para si pelo preço estimado - Vrerkaufermächtigung, bei welcher der Empfänger Selbstkaufrechet zum Schätzunspreize erhält.

Para Mayns, esse contrato se forma "quand je remets à un autre une chose qu'il la vende et m'en procure un prix. déterminé, ou bien qu'il me restitue au cas qu'il ne la vende pas (5).

Confrontando-se as diversas noções que nos ministram autorizados romanistas, desume-se o esfôrço empregado para traduzir em forma eclética a marcante concisão latina (6).

Perozzi inclue na definição a questão da propriedade da cousa entregue, salientando que o tradente não se despojou do domínio. "Se alcuno ad altri una cosa stimate perchè la venda a che prezzo vuole, a patto da renda il prezzo di stima o, se non l'ha venduta, renda la cosa, delle quale il tradente ha conservato il dominio (7).

RoNGA tambem o define: è quel contratto per cui taluno dà ad un altro una cosa affinchè la venda ad un prezzo determinato, coll'obbligo di restituere la cosa stessa al tradente ol di pagargli quel prezzo (8):

Thaller, em estudo a propósito da convenção d'aestimaifum, depois de considera-la como sendo um contrato que o Eidto escolheu como tipo, assim descreve o mecanismo da operação correspondente: “En quoi consiste l'opération? Res aestimata vendendla datur. Celui qui la reçoit compte la vendre. Mais il se peut qu'aucun acquéreur ne se présente, qu'il n'arrive pas à s'en défaire. Ipsam rem debit incorruptam reddere, aut aestimationem de qua convenit. Le contrat laisse donc au preneur de la marchandi-

(4) Enciclopedia de PaOLY e Wisswoa, verb aestimatorius contractus. Mackeldey - C. D. Romano, Lend. e Ricci, \& 198; Bonfante, Instituzioni, $\S 167$; Serafini, Instituzioni, Girard, Manuel de Droit Romaine, pg. 593.

(5) Cours, II, $\S 244$, p. 343.

(6) Bonfanti.

(7) Istituzioni, II, § 178.

(8) Istituzioni, II, § 176. 
se, dans un délai tantôt préfixé et tantô laissé à bonne foi des parties, l'alternative entre la restituition de l'objet ou le payment d'une somme convenue que nous appellerons, pour tout simplier, un prix" (9).

2 - E' de tradição afirmar-se que os Romanos não tinham predileção pelo comércio, que o destestavam, julgando indígnos os que ao tráfico mercantil consagravam a sua atividade. E' conhecida a sentença de CícERo: "nihil ingenuum potest habere officina, nolo eumdum populum imperatorem esse terrae ac portilorem", como tambem a apóstrofe de Vergílio, no livro VI da Eneida:

Excudent alii spirantia molli. aera, etc.

Tu regere imperio populus, Romane, memento, Hae tibi erunt Artes

Mas, si julgaram o comércio uma profissão inferior, não desdenharam os lucros que êle proporciona; não o faziam por si, mas entregavam-se às especulações por intermédio de seus escravos e de seus libertos. Desse costume é que surgiram as ações institória e exercitória, tão conhecidas, bem como o instituto da representação, e os demais institutos que nos herdaram.

Entre êles, encontramos o contrato estimatório, que surgiu em Roma, na afirmação de Costa "non appena assusero in Roma largo sviluppo i commerci e vi si avanzó il costum dei grandi commercianti di affidare ad altre a vendere al minuto parte delle mercanzie raccolta nei loro magazzine.

Uns situam o aparecimento do contrato já no $60^{\circ}$ século de Roma, com fundamento no testemunho de Plauto.

Lucrum ingens facto, praeterquam mihi meus pater

Debit aestimatas merceis: ita peculium

Conficio grande (10).

(9) Mélanges Ch. Appleton, pg. 645.

(10) MercatoR, 1, 1, 94-96. 
Mas, provavelmente, só se delineou no ocaso da República ou nos albores do Império. E' o que sustentam De Medio e Costa, secundados por Bertolini.

3 - Os elementos para a definição do contrato fornece-nos Ulpiano no fr. $1, \S 1$, Dig. 19.3, que transladamos:

"Aestimatio autem periculum facil enim, qui suscepit; aut igitur ipsam rem debebit incorruptam reddere, aut aestimationem, de qua convenit, bem como nesta passagem do fr. 13, Dig. 19.5: "Si tibi rem vendendam certo pretio dedissem ut quo pluris viendidisses, tibi haberes.

Com eles podemos apresentar uma definição que BoNFANTE julga ser a que mais se aproxima desses fragmentos, numa fórmula breve e concisa: "il tradens deposita presso l'accipiens una cosa stimata per la vendita, col-patto che costui renda o la stima o la cosa".

Verdade é que algumas definições, com fundamento no fr. $17, \S 1$, Dig. 19.5, se referem tambem ao preço $-\mathrm{Si}$ margarita tibí aestimata dedero, ut aut eadem mihi afferres, aut prestium eorum - como Paulo já nas suas sentenças referida - si rem aestimata tibi debero ut, ea distracta, pretium ad me differes - Si margarita tibi vendenda dedero, ut, si ea decem vendidisses, redderes mihi decem, si plures quod excedit, tu haberes (11).

Para Coviello, nestes últimos casos, não ha, na realidade, um contrato estimatório, que este se caracteriza pela obrigação em que fica o accipiens de restituir a cousa ou a estimação "redderes rem aut aestimationem", ma piuttosto un mandatum de vendendo accompagnato da deposito della cosia stimatia.

O quie caracteriza o contrato é a estima, sendo a aestimatio, no dizer de BoLAfFio, a contraprestação dia cousa nâo restituida. A referêncila ao preço, desde que este não coincida com a estimla, préviamente convencionada, desvirtua o contrato, rompendo a sula reuritimia.

(11) fr. 17, § 1, Dig. 19.5. 
Porque da sua essisência é a altternativa quie se contém nos primeiros textlols de Ulpiano. E' ela quem lhe traça a fisionomia e marca a individualidade.

4 - Do que acabamos de expor, examinando-se os elementos compendiados nas definições apresentadas, verificase que delas emiergem as notas esistenciais do contrato, assim relacionadas por BERTOLINI:

1 - Entrega da cousa parra ser vendida (rem vendendum) (12).

2 - Avaliaçãod da cousa entregule (certo pretio) (13).

3 - Obrigação alternativa para o accipiens de pagar o preço predeterminado ou restituir a cousa (ut aut eadem mihi adferres aut pretium) (14).

4 - Direito do accipiens de reter a diferença entre a aestimatio te o preço da venda. (ut, quo plunes vendidisses tibi haberes) (15).

Plara Brivs quatro são, tambem, as notas essênciais deste contrato,

1) die Hingabe zum Verkaufe

2) die Feststellung eines Auschlages (aestimatio)

3) die Alternativitát der Verplichtung zwischen der Zahlung der Auschlages und der Rückgabe der Ware

4) das Recht der Empfängers aut den Meheerlös (16).

(12) L. 13. pr. D. de praescr. verbis, 19.5 .

(13) Idem.

(14) L. 17, § 1, D. h. t.

(15) L. 13, p. D. de praescr. verbis, 19.5 .

(16) Frans Lipp - Beitrag zur Lehre vom Trödel contrat, p. 10. 
5 - Autores existem que não julgam essencial a primeira nota característica, reduzindo-a à entrega pura e simples. Mas tal redução vai de encontro às expressões que as fontes consígnam. Nelas se encontram a palavra res qualificada, e o fim da entrega esclarecido - vendenda, ut distracta. A entrega tem um fim desejaado pelos contraentes, que não miram simplles guarda ou diepósito: a venda e, na sua falta, a restitulição.

Não se concebe o contriato sem a estima prévia do valor da cousa entregue, porque esta lhe é base e fundamento, no dizer de Bertolini. Basta verificar os textos fundamentais

rem aestimatam - Paulo, 2.4.4

res aestimata vendenda datur — L. I. D. de aestimatoria, 19.3

aut aestimationem de qua convienit - L. I. D. de est., 19.3

si tibi rem vendendam certo pretio dedissem, sed sibi certum pretium excepit - L. 17, § 17, § 1, D. de praescr. verbis, $\mathbf{1 9 . 5}$

Si o preço não é marcado, não ha prévia estima, não ha o contratio especializado, o que acontece mesmo quando se dispuzesse que a cousa entregule deveria ser venidida pelo maior preço que alcançasse. "Il tradens, sustenta Bertolini, vuole realizzare un certo valone della cosa sua e perció la dà da venidere ad un altro, il quale la riceve con tale intendìmento e conformel volontà. Non vale certo opporre, come si è fato, che un a'estimatio, si presenta o può presentarsi in altri contratti; mentre poi è errato dire, come pure fu fatto, che il contratto stimatorio sussisterebbe anche quandlo si platutisse che l'accipienite dovesse vendere la cosa al maggior prezzo possibile" (17).

Houve ainda quem duvidaisse do terceiro requisito, sustentando que deixaria de haver a alternativa, si o accipiens

(17) op. cit. § 15. 
pagasse o preço no áto de recebê-la. Mas nesse caso, não haveria tão pouco o contrato estimatorio. Porque o accipiente, pagando o preço, adquire desde logo a propriedade d'a cousa, não passando a transação de uma venida pura e simles.

DE MÉDIo julga aindia que o último requisito não é essencial e BERToLıNı não põie dúvida em acompanhá-lo, não porque nãlo reconheça no accipiente o direito de reter a diferença, si houver, porque esse direito é certamente seguro e incontestavel, mas porque se trata de uma consequência dos limites dientro dos quails se cincunscreve a obrigação do accipilente de pagar a aestimatio ou restituir o objéto. "Ma, badiamo bene, observa o romanista, non alla cosa in sè, che il diritto dell'accipiens all'evientuale accedenza sulla aestimatio è certamente sicuro ed incontestabile: bensi alla cosia come eliemento essenziale o requisito del negozio; mentre non trattarsi che di una consequenza dei limiti dentro cui è circossoritta l'obbligazione sudetta dell'accipiente (prestare l'aestimiatio o rendere l'oggetto).

6 - 0 contrato estimatório apresenta grande afinidade com vários contratos nominadoss. Por fôrça da estima, muito se assemelha à venda; em razão do serviço prestado, póde ser considerado como locação, ou como mandato. $\mathrm{E}$ ainda era possivel divisiar-se nele uma espécie de sociedade (18). Será um contrato autônomo?

Daí o ter surgido, entre os antigos juriscionsultos, grande disputa em relação à ação a ser concedida para a sua execução.

DE Médio assinala a controvérsia como um ponto de especial importância, mas termina por considerá-lo, consioante a opinião generalisada um contrato autônomo. $O$ contrato é, na realidade, diferente dos outros em muitos passos (19).

Nãlo é venda, porque aquele que recebe uma cousa estimada para vender, não é obrigado a pagar um preço e

(18) Vinnio, op. cit .

(19) VInNio, op. cit. 
póde, si quizer, restituir a cousa, si não a vender (20). Na venda pura e simplles, não é possivel pensar seriamente. Basta pensar na possibilidalde da restituição da cousa. No contrato estimatório, a alternativa: a cousa ou a estima. $\mathrm{Na}$ venda o pagamento dio preço.

Quando recebe a cousa pana vender, não tem em mente o accipiens adquirí-la, pondo a mira tão sómente em obter um lucro, vendendo-a por um preço maior que o estimado. $E$ ' verdade que lhe é facultado ficar com a cousa, pagando a aestimatio. Mas não é o casso comum. Além do que, observa DE MÉdio, trata-sie de uma intenção posterior à conclusão do contrato ou subsidiária si dela contemporânea.

Thaller sustenta que se trata de uma venda, mas de uma venda e compra condicionada ,embora julgue a questão embaraçante "par ce que, d'une part, la livraison immediate de la marchandise au preneur suggère la pensée d'une vente exigibile et seulement résoluble, tandis que le renvoi du paiement à la date où ce preneur prendra parti fait croire plutôt à une vente jusque là suspendu (21). Thaller não se associa aos escrupulos de Ulpiano, julgando que a aproximação da operação com a venda é tão discutivel como com a locação ou com o mandato. O comercialista francês julga que se teria dado ao aestimatum maior relêvo en lui communiquant très franchement le caractère de la vente. (22).

Mas essa doutrina, no dizer de Bertolini, manifesta-se debil e imperfeita pela incerteza e discórdia entre os autores no determinarem o caráter e o conteúdo da condição (23).

(20) Jousserandot - L'ÉDIT Perpetuel.

(21) Op. cit., 652.

(22) Idem, pg. 651.

(23) "Quest'analisi della diversa intenzione o volontà contratuale nei due negozi, la compravendita ed il nostro, è sufficiente ad escludere puranco che il contratto stimatorio sia una vendita condizionatta; doutrina la quale, sebbene vanti qualche seguace autorevole, si manifesta debole e imperfetta già per l'incertezza e discordia degli scrittori nel determinare il carattere ed il contenuto della condizione" (II Cont. est., § 15). 
Para DE MÉbio, guia seguro para distinguir os dois contratos, é a intenção dos contraêntes: "L'intenzione dei contraenti ci deve essere di guida sicura nella disanima, ed è sempre il loro volere, desunto dalle circonstanze di fatto che accompagnano la concluzione del negozio giuridico, che distingue parimenti il contratto stimatorio da venda sottoposta a condizione sospensiva o resolutiva (24).

7 - Não se trata de locação. Na locação ,são elementos essenciais, de um lado, operae ou opus; doutro lado um certo salário - merces constituta. Mas no contrato estimatorio, póde faltar o primeiro elemento, de vez que o accipiens não é obrigado a vender e a entregar a aestimatio.

$\mathrm{O}$ segundo elemento tambem póde faltar. E' certo que, é possivel convencionar uma compensação, sem que se desnature o contrato. Além da diferença entre a aestimatio e o preço da venda, é possivel acrescentar-se ou estatuir uma compensação fixa: Daneben kam ihm eine feste Vergütung billigt sein (25).

\section{Dia mesma opinião é VinNio.}

Jousserandot sustenta quie a palavra utilis não significa que esta ação é dada uțiliter, embora um dos contratantes receba um salário, mas que se póde recorrer a ela em logar de empregar a ação de locação, a despeito do salário convencionado, "parce que cette convention diffère du luoage, en ce que celui qui est chargé de venidrie la chose a la faculté de la rendre ou sa valeur estimée" (26).

A fixação do salário é estranha à essência do contrato. Nem se diga que a diferença entre a aestimátio e o preço equivale ao salário na locatio, porque aquela é aleatória e incerta, ao passo que a retribuição, na locatio é essencial e certa (27).

(24) Op. cit., pg. 35 .

(25) Dernburg, Römisches Recht, com fundamento no fr. 2.

D. 19.3 Haec actio utilis est et si merces intervenit.

(26) Op. cit. pg.......

(27) BERTOLINI, op. cit., 408. 
BARASSI sustenta que se não trata de um contrato de trabalho: "Un altro caso in cui la prestazione di lavoro è successiva alla stipulazione del contratto, mà obbligatoria, è quello che riguarda il debitore nel contratto stimatorio che presuppongo contenga anche la promessa di una mercede. In fatti chi promette di restituir o la cosa o il suo valore in una somma determinata, si la vende, non si assume affato di venderla: l'attività della vendita qui è solo in condizione. Non vi è dunque contratto di lavoro" (28).

8 - Não é sociedade. A sociedade pressupõe outros elementos que se não encontram no contrato estimatório, falta neste o animus contrahendiae societatis e é esse elemento subjetivo que concorre para distinguir os dois contratos.

Jousserandot sustenta que não é sociedade porque o lucro é para aquele que se encarrega de vender o objéto, e, portanto, sòmente para uma das partes (29).

São de Ulpraño estas palavras — "et societas non videtur contracta in eo, que te non admisit socium distractionis, sed sibi certum pretium excepit" (30).

Verdade é que, no L. 44 D. pro socio, 17, 2, Ulpiano faz alusão à socidade, parecendo haver contradição entre os dois textos. Tal não ha, porém, porque, nesta passagem justamente se encontra o critério para diferenciar os dois contratos, o elemento subjetivo do animus contrahendae societatis.

A ação será pro soctio, si tiver havido a intenção de formar sociedade, caso contrario será a praescrciptis verbis que é a peculiar ao contrato estimatório.

Coviello assinalou, de modo claro e perfeito, a distinção: "Il fatto, base materiale del rapporto giuridico è il medesimo: ti do una cosa da vendere per vendere per un prezzo determinato. La consequenza ultima a cui si vuol giungere è sempre la stessa: io voglio il prezzo di stima, tu

(28) Contratto di Lavoro. I, 406.

(29) Op. cit., pg.. ...

(30) L. 13, fr. D. de praescr. verbis, 19.5. 
potrai approfitarvi del di più ritratto della vendita. Però in un caso ho intenzione di stringere teco cosi una società, nell'altro no. Qual è l'effetto di questa diversa intenzione? 1..$^{\circ}$ Se voglio stringere una società e tu t'accordi in questo con me, bisogna che tu ed io vogliamo anche tutte le consequenze che naturalmente derivano da tale specifica comune volontà. Ora, se in forza dell'intenzione di far società insieme, io son costretto a mettere in comune la res vendendia tu devi metterci di tuo la tua abilità, la tua industria: altrimenti non vi avrebbe società. Tu dunque sei obbligato a far di tutti per vendere. 2. ${ }^{\circ}$ ) Ma quando non havvi quell'intenzione, l'accipiens non ha altro obbligo che o di restituire la cosa o pagare il prezzo di stima, a sua scelta, egli non é obbligato ad impiegare la sua attività; dunque non é obbligato a metter nulla in comune; dunque non v'é società. Abbiamo trovato cosi la differenza caratteristica tra società e contratto stimatorio, e in base appunto all'intenzione dei contraenti. La differenza è che, nel contratto stimatorio l'obbligato ha la facoltà di concludere il contratto di compra-vendita o di astenerse restituendo la cosa; il che non può seguire si è voluto fare una società, poichè devendo esserci sempre la messa sociale, sia pure in attività personale, questa manca, acordandosi quella facoltà. Dunque il contratto stimatorio si distingue essencialmente della società" (31).

Tambem Bolafio não admite no contrato estimatório uma relação de sociedade: "Neanche, fra il tradens e l'accipiens, si stringe un rapporto sociale. Riprenda la cosa oppure riscuota la stima fissata preventivamente, il tradens, come regola, non partecipa al profitto della revendita (quod pluris vendidisses tibi haberes). Nè a sua volta l'accipiens è socio di opera o di industria: perchè, come fù rilevato, non è obbligato, mancvando un patto contrario, a spiegare una attività procacciante. Mal grado ciò, il sopropezzo è esclusivamente suo pel contratto originario: e la inesistenza

(31) Op. cit., pg. 415-416. 
di un vincolo sociale è poi esclusa dalla facoltà, non restringibile, dell'accipiens, di restituire, in ogni momento, ad libitum la cosa ricevuta" (32).

9 - Não se confunde outrossim, com o mandato. 0 mandato no Direito Romano carateriza-se pela gratuidade. $E^{\prime}$ na definição de Dernburg, a assunção de um encargo, sem recompensa (33). "Mandatum nisi gratuitum nullum est; nam originem ex officio atque amicia trahit, contrarium ergo est officio merces: interveniente. enim pecunia res ad locationem et condictionem potius respicit" (34). "Nec lucrum tibi ex hac causa adquirere debes, cum mandatum gratuitum esse debet" (35).

No contrato estimatório, como já vimos, é possivel pactuar-se uma retribuição, o que é incompativel com o mandato.

Tambem mais rigorosa é a posição do accipiens, e mais leve a do mandatario. Na realidade, observa DE MéDıo, "mentri questi si libera da ogni obbligo col gerire conscienziosamente l'affare oggetto del mandato, quegli invece deve sempre rispondere dell'aestimatio, a meno che non voglia restituere la cosa, anche quando abbia agito con buona volontà e rettitudine per riuscere nell escopo. Nè carrebbe obbiettare che il maggior rigore sfuma se egli può restituire la cosa, giacchè è da considerare che la facoltà della restituzione rapresenta appunto l'elemento moderatore introdotto per attenuare la grave responsabilità, la quale esiste di per si".

E' ainda dever do mandatário entregar ao mandante todos os proventos obtidos em consequência do mandato. "Ex mandato apud eum, qui mandatum suscepit, nihil remanere oportet, sicuti nem damnum pati debet si exigere faeneratam pecuniam non potuit" (36).

(32) BoLafio, Il contratto stimatorio, quale atto oggetivamente commerciale, n..$^{\circ} 5$.

(33) op. cit., § 372 .

(34) fr. $1, \S 4$ D. 19.1 Paulus, libro 32 ad edictum.

(35) fr. 36, § 1, D. eod Javolenus, libro 7 ex Cassio.

(36) fr. 20, D. 17,1 - Dernburg, § 373. 
O accipiens, no entanto, lucra o que alcançar além da importância prefixada: o que exceder lhe pertence. Não lhe cabe restituir o que receber a mais, na venda que fizer, e a tanto seria obrigado si fosse um mandatário.

Mayns sustenta que o contrato se assemelha à operação que hodiernamente se denomina comissão, opinião que é, tambem a de CoElho dA Rochit, quando sustenta que a condição aposta à entrega importa numa esplecie de comissão (37).

Bolafio refuta similhante opinião porque o accipiens não vende sòmente no próprio nome, mas tambem por conta própria, a cousa recebida, e a diferença entre a estima e o preço obtido pertence-lhe inteiramente: "L'Accipiens non vende soltanto in nome proprio, mà per conto proprio la cosa ricevuta: la differenza in più tra l'aestimatio e il prezzo di rivendita è a sua vantaggio esclusivo" (38).

Cuovis Bevilaqua, estudando o contrato, vê nele alguma cousa dé similhante à consignação (39). A similhança é possivel. Mas o contrato estimatório não se confunde com a comissão porque o accipiens não é obrigado a prestar contas do preço obtido, mas tão sòmente pagar a aestimatio.

10 - Essas divergências no apreciar a natureza jurídica do negócio em apreço, não podendo identificá-lo com os contratos nominados, pelos desvios que os incompatibilizam, deram em resultado a publicação de um edito em que o pretor dava, para tal negócio, uma ação especial — "Si res aestimata vendenda data fuerit, nec ipsa res incorrupta reddita sit, de aestimato actionem dabo".

Estavam negócios que tais munidos de ação, e, portanto, erigidos em contrato independente, subsistindo por si só,

(37) Maxns, II, 244, Il ressemble à l'opération que nous designons aujourd'hui par le nom de commission. - CoelHo DA RoCHA, D. Civil, II, \& 695 . Esta cláusula importa numa espécie de comissã̃o.

(38) op. cit., n.o 6.

(39) Dto. das Obrigações, § 120. 
apresentando analogias com os contratos nominados, mas separando-se deles por caracteristicas inconfundiveis.

Que o fim da instituição consignada no Edito foi o de cortar a dúvida que se suscitava dí-lo Ulpiano: "Actio de aestimato proponitur tollendae dubitationis gratia. Fuit enim magis debitatum, quum res aestimata vendenda datur, utrum ex venditio sit actio propter aestimationem, an ex locato, quasi rem vendendam locasse videas, an ex conducto, quasi operas conduxissem, an mandati" (40).

Assim, diz Petit, depois de terem procurado assimilar esta convenção a uma venda, a uma locação, a um mandato, acabaram os romanos por ver nela um contrato inominado, sancionado pela ação praescriptis verbis que tomou, nesse caso, a denominação particular de ação estimatória (41).

11 - "On hésitat pour savoir si cette convention constituait une vente, un louage, ou un mandat. On s'est tiré d'affaires en donnant l'action civilis in factum qui apparait dans la fonction primitive pour laquelle elle fut inventée, en même temps qu'ellesactionne comme premier contract inommé une convention que, la propriété n'étant pas transféré, ne rentre ni dans la combinaison do ut facias".

Um tal contrato não é venda, não é locação nem mandato, diz Dernburg. Por isso o pretor condescendeu em estabelecer 'para ele uma ação particular ex finı bona', a aestimatoria praescriptis verbis, por meio da qual se podia reclamar do revendedor a cousa ou o preço. "Ein derartiger Vertrag ist weder Kauf, noch Miete, noch Mandat. Daher verstand sich der Prätor dazu für denselben eine ergene Klage "ex fide bona" aufzustellen, die aestimatoria praescriptis verbis, mittels deren der Geber di Sache oder den Preis won Trödler einfordern Konnte" (42).

(40) L. 1, D. de aest., 19,3.

(41) Traité Élémentaire, n. ${ }^{\circ} 408$, pg. 416.

(42) Vol. II, § 7; pg.. .. 
Foi Karlowa quem primeiro observou que a actio aestimatoria foi o ponto de partida para os contratos inominados, "und dass eine allgemeine für alle bezúglichen Fälle bestimmte actio praescriptis verbis nicht im prätorischen Edikte proponiert war" (43).

Lenel, segundo Dernbura, deu amplo desenvolvimento a este conceito.

12 - Caracterizada a natureza jurídica do contrato, em face dos textos romanos, e assinalada a ação que the foi atribuida, não se esgota a série de dificuldades. $E$ surgem, a propósito, problemas os mais interessantes. Na verdade, a passagem do Digesto, que se reporta ao contrato em exame é fonte de perenes controvérsias. Desde o tempo dos glosadores que tal acontece assinala Frans LIPP, na obra já referida.

1 - E' um contrato real ou consensual?

2 - Na dúvida presumir-se-à um contrato estimatório?

3 - Quem é o proprietário?

4 - Responde o accipiens pelo casus?

5 - Que se deve pensar da chamada aestimatoria praescripti verbis.

Resolvidas as 4 primeiras questões, com o auxílio de contribuição que, para sua solução, trouxeram SEuffer, EDwrd, Chambon, Brinz, BekKer e outros, continua o precitado monografista, so erscheint dagegen die Frage: Wer trägt beim sogenannten Trödelcontract die Gefahr? und die eng damit verbundene Controverse darübert;

Wie die sogenannte aestimatoria praescriptis verbis actio zu denken sei (44).

E, a respeito desta última, LIPP contraria a opinião geralmente aceita, de que se trata de um contrato inominado, e repele a atribuição de uma actio praescriptis verbis, fundado, principalmente, na dissertação de Störcke,

(43) Dernberg - op. cit., nota 15 em $\$ 263$.

(44) F. LIPP, op. cit., pg. 11. 
(dissertatio de contractu aestimatorio tanquam contractu nominato HaLAE 1756), no exame dos textos, e em confronto do texto latino com o correspondente nas Basilicas, por não encontrar neles refierência alguma àquelas palavras: praescriptis verbis: "gegen ihre Gültigkeit wenden sich feindlich alle Gründe, welche direkt oder indirekt für die "aestimatoria" überhaupt sprechen" (45).

$\mathrm{E}$, depois do confronto referido, conclúe: Hier ist sowol im griechischen als auch im lateinischen Texte weit und breit nichts von einer actio prxescriptis verbis zu sehen: die Klage, welche hier gegeben, ist die aus dem Trödelcontrat schlechtweg, actio de aestimato" (46). A tese, que vai ao arrepio da sustentada pela maioria dos románistas, merece minudente exame, para o qual muito poderão concorrer as pesquisas de GRADENwitz e de LENEL, aquele no seu célebre estudo sôbre as interpolações (Interpolationem in dem Pandekten) e este em estudo publicado na "Zeitschrf, der Sav. Stiftg. Abt. Bd-9, S. 181", consoante de Dernburg (47). Gradenwitz demonstrou que esta actio praescriptis verbis procede da jurisprudência bisantina, de onde foi transplantada para o Corpus Juris, mediante interpolação, pelos compiladores. Os juristas clássicos não conheciam uma "actio" praescriptis verbis - precisamente o" característico destes casos é a ausência de uma ação específica adatavel - mas uma simples praescriptis verbis "ag?$r e$ ", uma forma geral de ação, aplicavel a casos os mais he-terogêneos (48).

(45) Idem, pg. 20.

(46) Idem, pg. 21.

(47) op. cit., pg. 550, nota 15 , in fine.

(48) Sorm, trad. esp. nota 1 a pg. 368. 